Classification

Physics Abstracts

$61.10-61.30-76.60$

\title{
Mesomorphic properties of short chains substituted heteroaromatic salts
}

\author{
M. Veber $\left({ }^{1,2}\right)$, P. Sotta $\left({ }^{1}\right)$, P. Davidson $\left({ }^{1}\right)$, A. M. Levelut $\left({ }^{1}\right)$, C. Jallabert $\left({ }^{2}\right)$ and \\ H. Strzelecka $\left({ }^{2}\right)$ \\ (1) Laboratoire de Physique des Solides (*), Bât. 510, Université Paris Sud, 91405 Orsay, France \\ (2) ESPCI $(* *), 10$ rue Vauquelin, 75231 Paris Cedex 05, France
}

(Reçu le 16 janvier 1990, révisé le 28 février 1990, accepté le 7 mars 1990)

\begin{abstract}
Résumé. - Deux tétrafluoroborates de 2,4,6-triaryl pyrylium substitués par 6 courtes chaînes alcoxy (éthyloxy: $\mathrm{C}_{2}$, propyloxy: $\mathrm{C}_{3}$ ) ont été synthétisés. Les méthodes classiques de caractérisation (DSC et microscopie optique) montrent que ces deux nouveaux composés présentent la même phase colonne $D_{h}$ que les autres membres de la série homologue $\left(C_{4}\right.$ à $\left.C_{12}\right)$. Toutefois, ces deux nouveaux composés sont sujets à décomposition chimique lorsqu'ils sont chauffés pendant quelques heures dans le domaine de température de la mésophase. L'existence d'une telle mésophase implique que les colonnes voisines sont décorrélées. Habituellement, on suppose que ce sont les chaînes aliphatiques fondues qui jouent ce rôle ce qui pose un problème dans le cas de chaînes aussi courtes que $\mathrm{C}_{2}$ ou $\mathrm{C}_{3}$. Dans ce cas, les anions $\mathrm{BF}_{4}^{-}$doivent participer à la formation de la mésophase. Afin d'obtenir des renseignements sur le comportement de ces anions, nous avons d'abord effectué une étude complète de la série homologue par diffraction des rayons X. A partir des intensités des réflexions du réseau bidimensionnel pour chaque composé de la série, nous avons obtenu un modèle simple de l'organisation moléculaire de la mésophase. Les $\mathrm{BF}_{4}^{-}$sont désordonnés mais situés proches des cœurs aromatiques des cations. Nous avons aussi effectué une étude par RMN du ${ }^{19} \mathrm{~F}$ des composés $\mathrm{C}_{4}, \mathrm{C}_{5}, \mathrm{C}_{8}$ et $\mathrm{C}_{12}$ qui montre que les ions $\mathrm{BF}_{4}^{-}$présentent plusieurs types de mouvements dans la mésophase. Ainsi, à haute température, le mouvement de réorientation sphérique des ions $\mathrm{BF}_{4}^{-}$se fait librement. Ce mouvement pourrait entraîner la décorrélation des colonnes voisines et donc l'apparition de la mésophase à haute température dans le cas des composés à courtes chaînes $\mathrm{C}_{2}$ et $\mathrm{C}_{3}$.
\end{abstract}

\begin{abstract}
The synthesis of triaryl-2,4,6 pyrylium tetrafluoroborates substituted by six short (ethyloxy: $\mathrm{C}_{2}$, propyloxy: $\mathrm{C}_{3}$ ) alcoxy chains has been performed. Classical characterization methods (DSC and optical microscopy) show that these two new compounds present the same columnar phase $D_{h}$ as that displayed by the other members of the homologous series $\left(C_{4}\right.$ to $\left.C_{12}\right)$. However, these two new compounds are subject to chemical decomposition when heated for a few hours in the mesophase domain of stability but close to $T_{\mathrm{c}}$. The existence of such a mesophase implies that neighbouring columns be discorrelated. Disordered alcoxy chains are usually assumed to play this role which is however questionable in the case of chains as short as $n=2$ or 3 . In this case, the $\mathrm{BF}_{4}^{-}$anions should play a role in the mesophase formation. In order to obtain information about the behaviour of these anions, we have first performed a comprehensive
\end{abstract}

$\left(^{*}\right)$ Associé au CNRS (URA D 0002).

$\left.{ }^{* *}\right)$ UA 429, Chimie et Electrochimie des Matériaux Moléculaires. 
$X$ ray diffraction study of the whole homologous series. From the intensities of the 2D lattice reflections for all members of the series, we derived a simple model of the molecular organization of this mesophase in which the $\mathrm{BF}_{4}^{-}$anions are spatially disordered but still located close to the aromatic cores of the cations. We also performed a ${ }^{19} \mathrm{~F}$ NMR study of compounds $\mathrm{C}_{4}, \mathrm{C}_{5}, \mathrm{C}_{8}$ and $\mathrm{C}_{12}$, which shows that the $\mathrm{BF}_{4}^{-}$anions undergo several types of motion in the mesophase. At high temperatures, the spherical reorientation motion of the $\mathrm{BF}_{4}^{-}$anions is completed. The onset of this motion may be mainly responsible for discorrelating neighbouring columns and therefore for inducing the $D_{h}$ phase displayed at high temperatures by compounds $C_{2}$ and $C_{3}$.

\section{Introduction.}

Up to now, when series of homologous discotic compounds could be synthetized, the columnar mesophases have generally been obtained for aliphatic chains at least as long as 4 or 5 methylene groups [1]. A few exceptions to this rule have appeared in the literature [2-5]. However, the natures and the organizations of these phases have not always been fully elucidated. In the course of our studies upon thermotropic liquid crystalline salts based on heteroaromatic cations, we have shown that triaryl-2,4,6 pyrylium tetrafluoroborates 1 with 6 alcoxy chains $\left(n=4,5,8,12\right.$ called respectively $C_{4}, C_{5}, C_{8}$ and $\left.C_{12}\right)$ display columnar phases $\left(D_{h}\right)$ on a wide range of temperature [6-8].

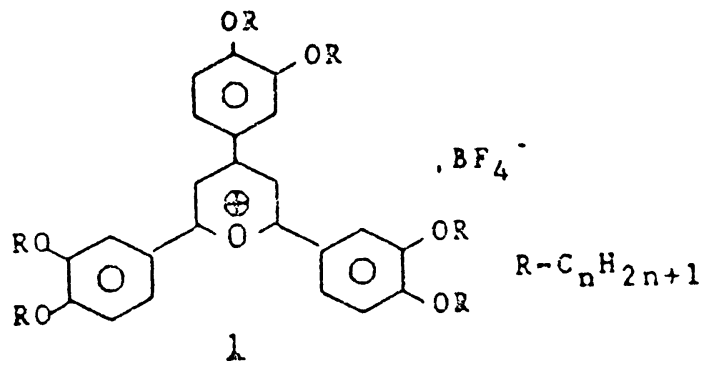

This series of compounds was likely to display a columnar mesophase in the case of short chain substituents because of the role of the anions. Indeed, the anions may participate in the loss of correlation of neighbouring columns. Therefore, in this context, we have synthesized the compounds 1 with $n=2$ and $3: \mathrm{C}_{2}$ and $\mathrm{C}_{3}$. (We had already synthesized compound 1 with $n=1, \mathrm{C}_{1}$. It only displays a crystalline phase [8]).

In the following, we show by the usual characterization methods that these two new compounds present the same $D_{h}$ mesophase as that displayed by the other members of the series. Then, we present a comprehensive X-ray diffraction study of all members of the homologous series and propose a simple model for the molecular organization in the mesophase. Finally, in order to try to understand the role of the anions in the mesophase formation and stability, we shall also present ${ }^{19} \mathrm{~F}$ NMR experiments on compounds $\mathrm{C}_{4}, \mathrm{C}_{5}$, $\mathrm{C}_{8}$ and $\mathrm{C}_{12}$ in the mesophase.

Tetrafluoroborates $\underline{1}$ were obtained by using a procedure already described [6; 8]. The purity of the new salts $\left(\mathrm{C}_{2}\right.$ and $\left.\mathrm{C}_{3}\right)$ has been checked by IR, ${ }^{1} \mathrm{H} \mathrm{NMR}$ and UV-vis spectroscopies.

\section{Characterization.}

1.1 MicrosCopic OBSERVATIONS. - They were performed with a Leitz Orthoplan microscope under polarized light. The variable temperature stage was a Mettler FP80. 
Both compounds present mesomorphic phases. On heating, an isotropic phase appears in equilibrium with the mesomorphic one. This is indicative of the presence of impurities either in the original sample or produced by decomposition. Some arguments will be given to choose between these two possibilities (see below).

1.1.1 Compound $\mathrm{C}_{2}$. - On the first heating $\left(10^{\circ} \mathrm{C} / \mathrm{min}\right)$ the transitions $\mathrm{K} \rightarrow \mathrm{M}$ and $\mathrm{M} \rightarrow \mathrm{I}$ are observed around 200 and $210-217^{\circ} \mathrm{C}$ respectively. No characteristic textures could be obtained. On slow cooling $\left(1^{\circ} \mathrm{C} / \mathrm{min}\right)$, either birefringent or homeotropic domains appear at $211^{\circ} \mathrm{C}$ growing from the isotropic phase. By further cooling, circular homeotropic germs turned to hexagons, then to digitated stars separated by the isotropic liquid (Figs. 1a, 1b).

These textures are characteristic of a columnar hexagonal phase $D_{h}$ [1]. It has not been possible to obtain the hexagonal phase pure, i.e. without the isotropic liquid. Crystallization occurs at $194^{\circ} \mathrm{C}$.

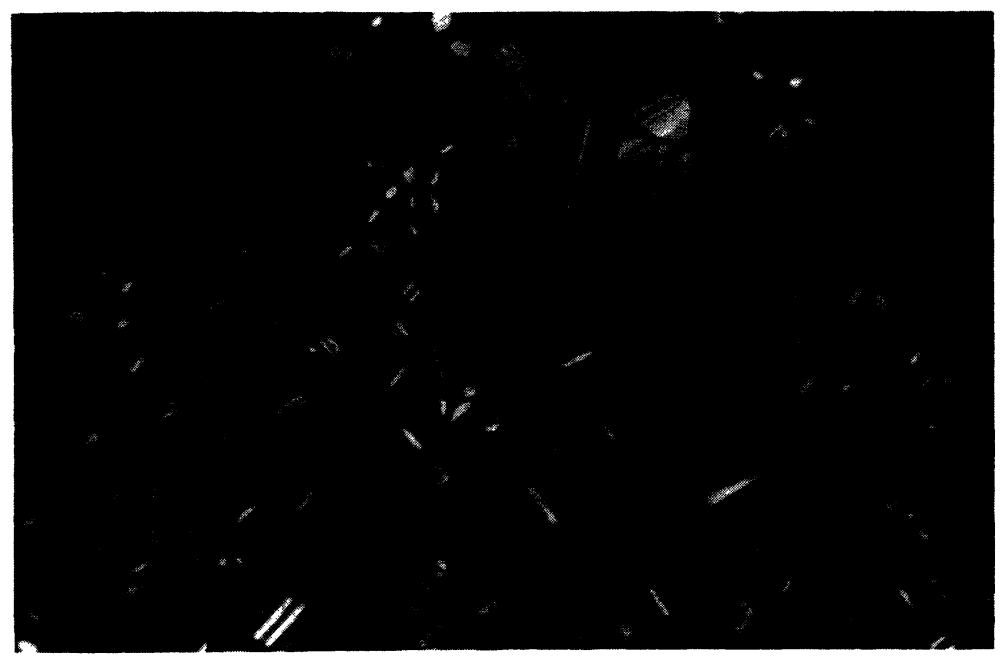

a)

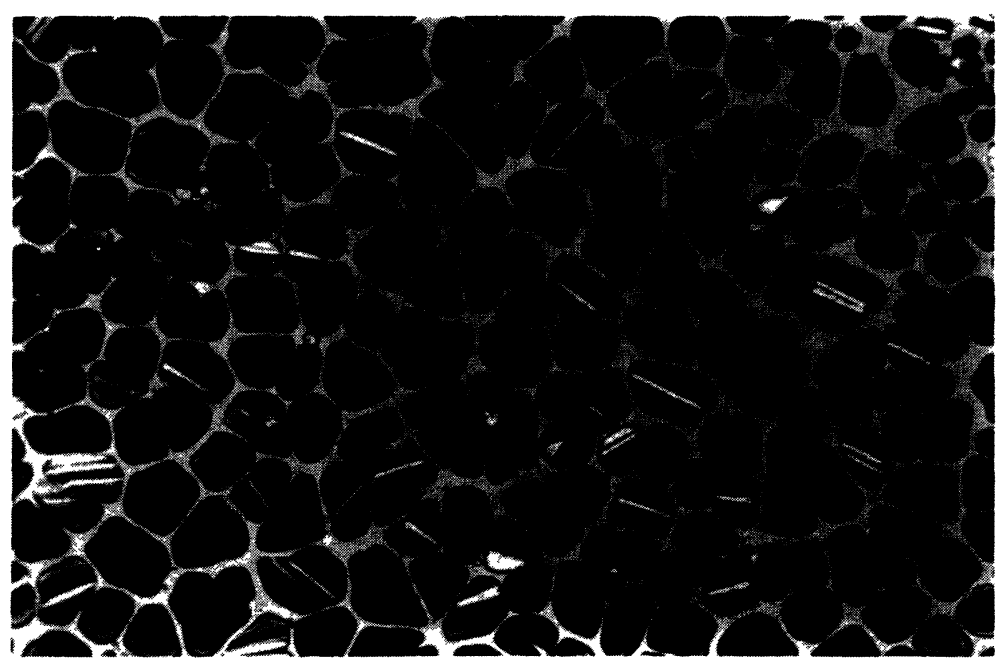

b)

Fig. 1. - (a) Texture of compound $\mathrm{C}_{2}$ at $207^{\circ} \mathrm{C}$. (b) Texture of compound $\mathrm{C}_{2}$ at $202^{\circ} \mathrm{C}$. 
1.1.2 Compound $\mathrm{C}_{3}$. - On the first heating, the transition $\mathrm{K} \rightarrow \mathrm{M}$ could not be accurately determined. Birefringent textures without any isotropic liquid could be observed around $175^{\circ} \mathrm{C}$.

Further heating leads to the clarification of the sample $\left(240^{\circ} \mathrm{C} ; 10^{\circ} \mathrm{C} / \mathrm{min}\right)$. Textures characteristic of an hexagonal columnar phase could be observed upon cooling (Figs. 2a and b) together with some isotropic liquid expelled from the hexagonal phase. The amount of this isotropic phase is a function of the temperature at which the sample has been heated. Moreover, if the sample is kept for a long time at high temperature, in the mesophase domain of stability, the amount of isotropic liquid increases.

These observations can be interpreted by a decomposition of the sample upon heating; more important in the case of short chains substituted salts. Indeed, in this case, the pyrylium

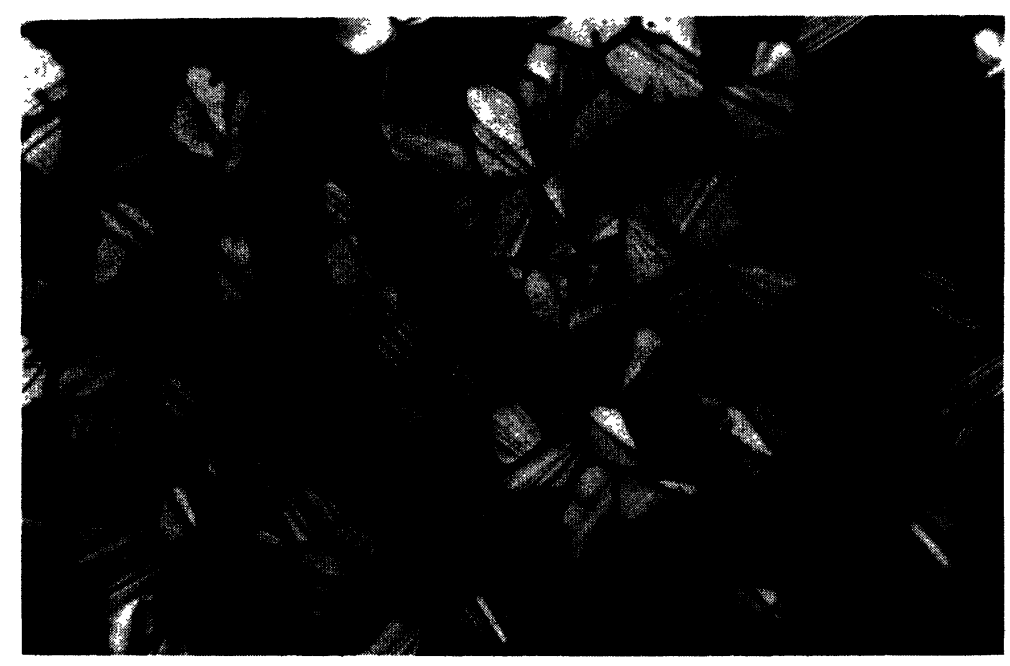

a)

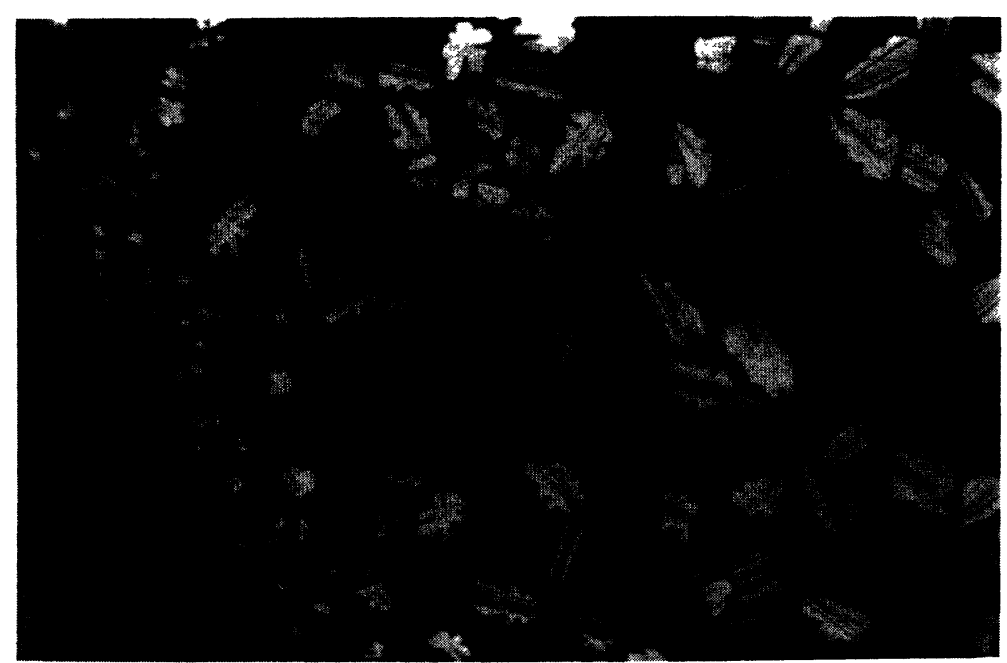

b)

Fig. 2. - (a) Texture of compound $\mathrm{C}_{3}$ observed on cooling at $150{ }^{\circ} \mathrm{C}$. (b) Texture of compound $\mathrm{C}_{3}$ observed on cooling at $148^{\circ} \mathrm{C}$. 
ring is less protected by a paraffinic surrounding and then more subject to chemical decomposition.

No crystallization is observed by further cooling indicating that the columnar phase can be frozen in at room temperature.

1.2 DSC. - DSC experiments were performed using a Mettler TA 3000 System.

In the case of compound $\mathrm{C}_{3}$, transitions are only observed on the first heating $\left(10^{\circ} \mathrm{C} / \mathrm{min}\right)$ and are the following:

$$
\mathrm{K}_{1} 70^{\circ} \mathrm{C} \rightarrow \mathrm{K}_{2} 167^{\circ} \mathrm{C} \rightarrow \mathrm{D}_{\mathrm{h}} 247^{\circ} \mathrm{C} \rightarrow \mathrm{I} .
$$

Upon subsequent cooling and heating cycles the transitions cannot be detected anymore.

In the case of compound $\mathrm{C}_{2}$, diagrams of the first heating and cooling cycle are shown in figure 3 (rate $10^{\circ} \mathrm{C} / \mathrm{min}$ ). After several cycles, a slight decrease of the $\mathbf{M} \rightarrow \mathrm{I}$ transition temperature is observed (approximately $5{ }^{\circ} \mathrm{C}$ for six cycles). The other transitions are not affected by repeated heatings. These observations are consistent with a slow decomposition of the sample induced by high temperatures. Table I summarizes the transition temperature measurements.



Fig. 3. - DSC diagram of compound $\mathrm{C}_{2}$ recorded at $10{ }^{\circ} \mathrm{C} / \mathrm{min}\left(\mathrm{K}_{1}, \mathrm{~K}_{2}\right.$ : crystals, $\mathrm{D}_{\mathrm{h}}$ : hexagonal columnar phase, I : isotropic phase).

Table I. - Transition temperatures measured by DSC and optical microscopy on heating.

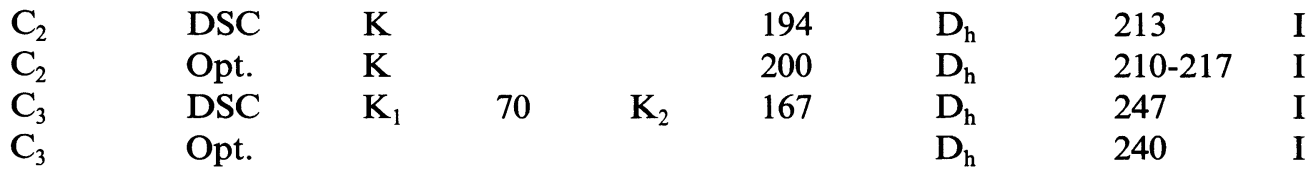

\section{2. $X$ ray diffraction.}

2.1 EXPERIMENTAL. - A Guinier powder camera has been used to measure accurately the structural parameters of the mesophases and a fixed film-fixed sample apparatus has been employed to probe the mesophase symmetry of aligned samples. 
First, let us describe the powder camera :

A monochromatic X-ray beam $\left(\lambda \mathrm{CuK} \alpha_{1}=1.5405 \AA\right)$ was obtained by reflection on a cylindrical quartz Johannson monochromator. The X-ray beam is in shape of a small vertical linear segment $\left(10 \times 0.1 \mathrm{~mm}^{2}\right)$. The resolution of the set up is $\Delta q=10^{-2} \AA^{-1}$ where $q$ is the momentum transfer vector $q=(4 \pi \sin \theta) / \lambda$ and $2 \theta$ is the scattering angle. The Guinier camera diameter was $114.7 \mathrm{~mm}$. The sample in its Lindemann capillary tube $(\phi=$ $1.0 \mathrm{~mm}$ ) rotates along its vertical axis and is heated by an air stream (temperature regulated to $\pm 2{ }^{\circ} \mathrm{C}$ ). Exposure times were typically $\approx 10$ hours [6].

The fixed film-fixed sample set-up uses a point focusing $\mathrm{X}$-ray beam $(\lambda \mathrm{CuK} \alpha=$ $1.541 \AA$ ) issued from a doubly bent pyrolitic graphite monochromator. The camera is evacuated in order to supress air scattering. The sample is held in an oven heated by an oil bath (temperature regulated to $\pm 1^{\circ} \mathrm{C}$ ) [6].

Aligned samples were obtained by spreading a small amount of product onto thin mica single crystal sheets with a spatula [6].

2.2 RESULTS. - Figure $4 \mathrm{a}$ shows the powder pattern displayed by compound $\mathrm{C}_{3}$ recorded at a temperature just above the $\mathrm{K}-\mathrm{D}_{\mathrm{h}}$ transition temperature in order to lessen the decomposition of the sample and figure $4 \mathrm{~b}$ is a scheme of this pattern. Let us describe figure $4 \mathrm{~b}:$ at small angles, several resolution limited reflections (a) can be seen. The corresponding lattice spacings are in ratios $1: \sqrt{3}: \sqrt{4}: \sqrt{7}$ which are characteristic of a $2 \mathrm{D}$ hexagonal lattice. The lattice parameter is $a=19.6 \pm 0.1 \AA$ at this temperature. At wide angles, a weak diffuse ring (b) can be detected around $s=1 / 4.5 \AA^{-1}$ where $s$ is the scattering vector $(|s|=$ $(2 \sin \theta) / \lambda)$. It corresponds to the interferences from the disordered parts of the molecule [6] : the small aliphatic chains and the $\mathrm{BF}_{4}^{-}$anions. Still at wide angles, an extra reflection (c) can be seen around $s=1 / 3.5 \AA^{-1}$. Though rather sharp, it is not resolution limited and classically corresponds to the stacking of the molecules inside the columns [6]. Such a powder pattern exhibiting only $2 \mathrm{D}$ long range positional correlations of the molecules is characteristic of a mesophase [9]. Thus it confirms the microscopic observations and the DSC investigations. In the case of compound $\mathrm{C}_{2}$, our powder set-up was not well adapted to the conditions of occurrence of the mesophase (too high temperature and too small temperature domain of stability). Anyway, figure 5 is an X-ray diffraction pattern of unoriented compound $\mathrm{C}_{2}$ in its mesomorphic state (at a temperature $T=205^{\circ} \mathrm{C}$ ) recorded with the fixed film-fixed sample

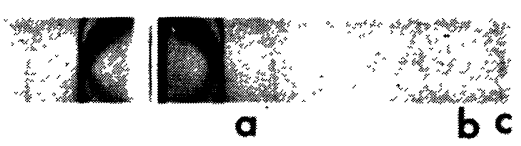

a)

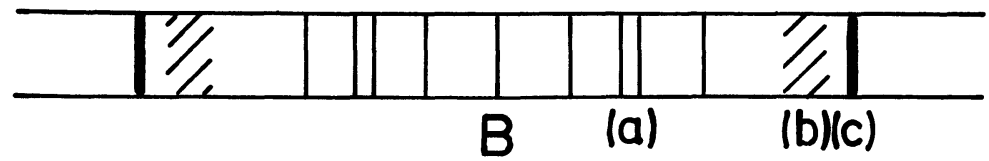

b)

Fig. 4. - (a) Powder pattern of compound $\mathrm{C}_{3}$ recorded with the Guinier camera. $\lambda \mathrm{CuK} \alpha_{1}=$ $1.5405 \AA$. Sample-film distance : $57 \mathrm{~mm}$. Temperature : $150{ }^{\circ} \mathrm{C}$. (b) Schematic representation of figure 4a : (a) Bragg reflections, (b) diffuse ring, (c) wide angle reflection. B beam trap. 


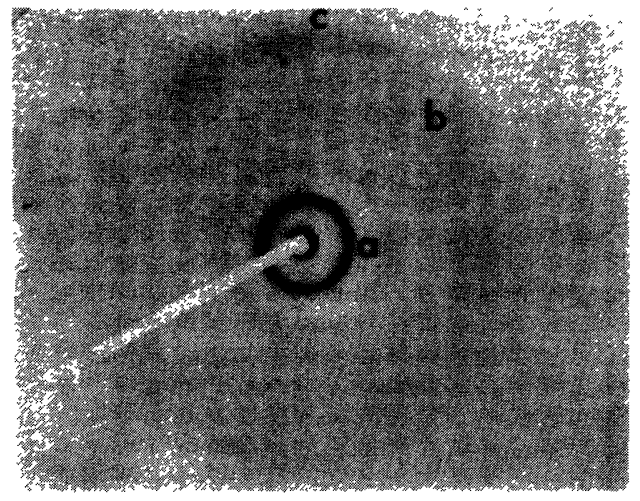

Fig. 5. - Powder pattern of compound $\mathrm{C}_{2}$ recorded with the fixed film-fixed sample set up. $\lambda \mathrm{CuK} \alpha=1.54 \AA$. Sample-film distance : $60 \mathrm{~mm}$. Temperature : $205^{\circ} \mathrm{C}:$ (a) Bragg reflections, (b) diffuse ring, (c) wide angle reflection.

apparatus. This pattern is qualitatively similar to that of figure 4a. It also presents, at small angles, the sharp reflections (a) of a $2 \mathrm{D}$ hexagonal lattice of parámeter $a=18.0 \pm$ $0.5 \AA$, at wide angles a diffuse ring (b) around $s=1 / 4.5 \AA^{-1}$ and a sharper reflection (c) around $s=1 / 3.5 \AA^{-1}$.

Figures $6 \mathrm{a}, \mathrm{b}$ show the $\mathrm{X}$-ray diffraction patterns of aligned samples of compounds $\mathrm{C}_{2}$ and $\mathrm{C}_{3}$. Figure $6 \mathrm{c}$ is a scheme of these patterns. (The meridian is the spreading direction $S$.) In the case of compound $\mathrm{C}_{2}$, the pattern is that of the mesophase quickly frozen in at room temperature just after spreading. In the case of compound $\mathrm{C}_{3}$, the pattern has been recorded on cooling, at $150^{\circ} \mathrm{C}$. From the location of the small angle reflections (a) along the equator, - we deduce that the $2 \mathrm{D}$ hexagonal lattice is perpendicular to the spreading direction. From the location of the outer reflection (c) along the meridian, we deduce that the columns are oriented along the spreading direction. The wide angle diffuse ring (b) can hardly be seen on these patterns but it seems to be slightly oriented (it is also superimposed on some thermal scattering due to the mica sheet). Since its intensity follows the form factor of the disordered parts of the molecule (anions and alcoxy chains), then these parts are slightly oriented by the spreading process. Therefore, the mesophase displayed by compounds $\mathrm{C}_{2}$ and $\mathrm{C}_{3}$ can be unambiguously identified as a columnar mesophase of the type $D_{h}$. (Space group : P6/m m m, one of the 80 planar space groups [10].) This mesophase type is the same as that displayed by the other members of the series [8]. The coherence length of the columnar stacking derived from the width of the wide angle reflection (c) is about $400 \AA$ for both compounds $C_{2}$ and $C_{3}$.

Since compounds $C_{2}$ and $C_{3}$ both display the same mesophase $D_{h}$ as the other members of the series, it is interesting to compare (Tab. II) the geometrical parameters of the $D_{h}$ phase

Table II. - Geometrical parameters of the $\mathrm{D}_{\mathrm{h}}$ phase for all the compounds of the series.

$\begin{array}{lccccccc} & T^{\mathrm{re}} & a(\AA) & c(\AA) & d\left(\mathrm{~g} \mathrm{~cm}^{-3}\right) & \ell(\AA) & L(\AA) & \ell / L \\ \mathrm{C}_{2} & - & - & - & - & - & - & - \\ \mathrm{C}_{3} & 205^{\circ} \mathrm{C} & 18.0 \pm 0.5 & 3.58 & 1.09 & 2.00 & 2.50 & 0.8 \\ \mathrm{C}_{4} & 150{ }^{\circ} \mathrm{C} & 19.6 \pm 0.1 & 3.47 & 1.07 & 2.80 & 3.75 & 0.75 \\ \mathrm{C}_{5} & 150{ }^{\circ} \mathrm{C} & 21.1 \pm 0.1 & 3.45 & 1.03 & 3.55 & 5.00 & 0.71 \\ \mathrm{C}_{8} & 150{ }^{\circ} \mathrm{C} & 22.8 \pm 0.1 & 3.40 & 0.99 & 4.40 & 6.25 & 0.70 \\ \mathrm{C}_{12} & 150^{\circ} \mathrm{C} & 26.9 \pm 0.1 & 3.40 & 0.91 & 6.45 & 10.0 & 0.65 \\ & 150{ }^{\circ} \mathrm{C} & 31.2 \pm 0.1 & 3.40 & 0.87 & 8.60 & 15.0 & 0.57\end{array}$






a)

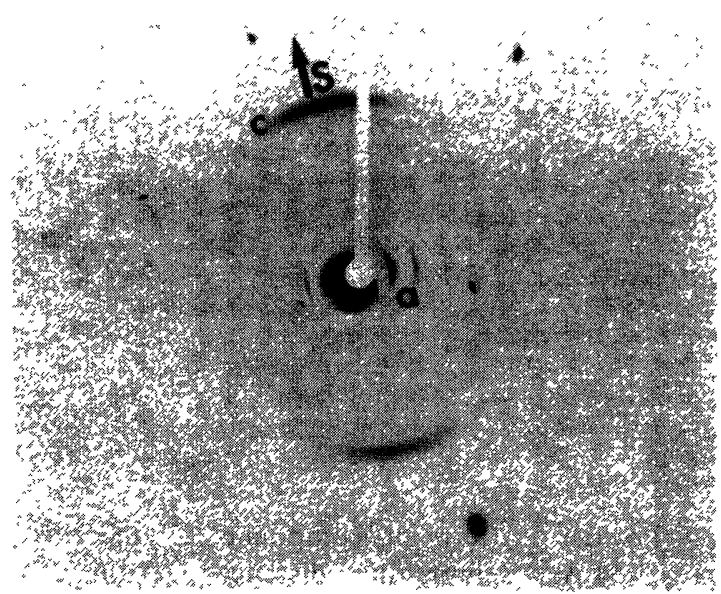

b)

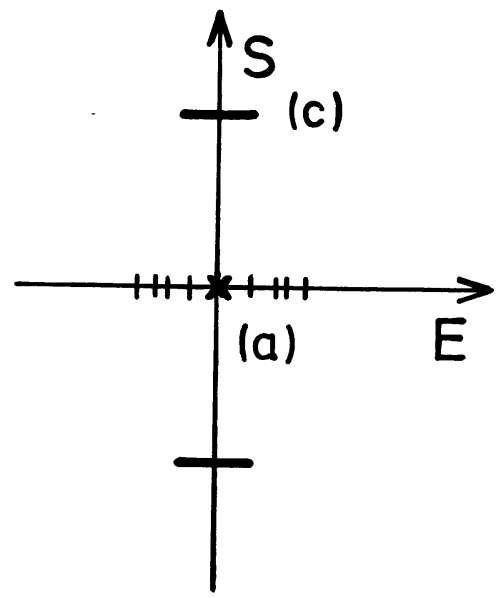

())

Fig. 6. - (a) X-ray diffraction patterns of an aligned sample of compound $\mathrm{C}_{2}$. S : spreading direction. Sample frozen in at room temperature just after spreading onto a mica sheet itself contained in a capillary. (The vertical lines are due to the edges of the capillary.). (b) X-ray diffraction pattern of an aligned sample of compound $\mathrm{C}_{3}$. $\mathrm{S}$ : spreading direction. Temperature : $T=150^{\circ} \mathrm{C}$. (c) Schematic representation of figures $6 \mathrm{a}, \mathrm{b}:$ (a) Bragg reflections, (c) wide angle reflection, $\mathrm{S}:$ spreading direction, meridian, E : equator. The slightly oriented diffuse ring (b) can hardly be seen on these patterns and is therefore not represented here (see text).

for all the compounds of the series : the hexagonal parameter $a$ and the stacking periodicity $c$ are directly measured on the powder X-ray diffraction patterns. The mesophase density is calculated thanks to the formula : $d=2 M /\left(N_{\mathrm{A}} \sqrt{3} a^{2} c\right)$ where $M$ is the molar mass and $N_{\mathrm{A}}$ is Avogadro's number. $\ell$ is the apparent chain length calculated from $2 \ell=a-$ $2 R$ where $R=7 \pm 0.5 \AA$ is the aromatic core radius measured on Dreiding stereomodels. $L$ is the trans-trans chain length also measured on Dreiding stereomodels.

Let us now plot the cell volume $V_{\mathrm{c}}=\sqrt{3} a^{2} c / 2$ versus the number of $\mathrm{CH}_{2}$ groups in the aliphatic chains (Fig. 7). One can see that, within the experimental errors, all points fall on a straight line, even for compounds $C_{2}$ and $C_{3}$. The fact that the points relative to compounds $\mathrm{C}_{2}$ and $\mathrm{C}_{3}$ are aligned with those of compounds $\mathrm{C}_{4}-\mathrm{C}_{12}$ shows that the sample degradation is 


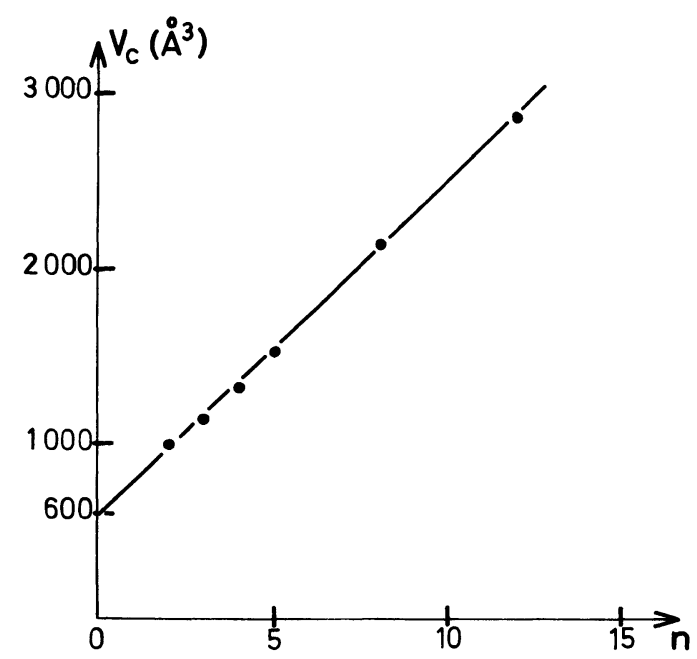

Fig. 7. - Volume cell $V_{\mathrm{c}}=\sqrt{3} a^{2} c$ versus the number $n$ of $\mathrm{CH}_{2}$ groups.

not too important during the time of the experiments. In particular, the $\mathrm{X}$ ray measurements are not hampered by the possible presence of a small amount of isotropic phase detected by optical microscopy. The slope of this straight line $\left(\approx 32 \AA^{3}\right.$ per $\mathrm{CH}_{2}$ group) represents the volume per methylene group. This volume is comparable to that found for other classical discotic compounds $[11,12]$. The intercept $\left(\approx 600 \AA^{3}\right)$ represents the volume of the non aliphatic part of the compound (i.e. the aromatic core and the $\mathrm{BF}_{4}^{-}$anion). Since the volume of the $\mathrm{BF}_{4}^{-}$anion is estimated to $\approx 75 \pm 10 \AA^{3}$ [13], we deduce the volume of the aromatic core $V_{\mathrm{ar}}=525 \AA^{3}$ and therefore an aromatic core radius of $R=6.95 \pm 0.10 \AA$ in agreement with that measured on Dreiding stereomodels $(7.0 \pm 0.5 \AA)$.

Now, since the mesophase displayed by all compounds seem not only to be of the same symmetry $\left(D_{h}\right)$ but also to present the same type of packing, it is tempting to propose a simple model for the molecular organization of this $D_{h}$ phase valid for all members of the series. Such a model may be derived by inspecting the intensity distribution of the different $2 \mathrm{D}$ lattice reflections for each compound. Table III shows the qualitative intensities of these reflections for all the members of the homologous series. These intensities have been directly estimated on powder patterns (except for $\mathrm{C}_{2}$ ) and corrected for the Lorentz-polarization and multiplicity factors.

Table III. - Intensities of the different 2D lattice reflections (directly estimated on powder patterns, except for $\mathrm{C}_{2}$, and corrected for the Lorentz-polarization and multiplicity factors) for each compound. VS : very strong, $\mathrm{S}:$ strong, $\mathrm{m}:$ medium, $\mathrm{w}:$ weak, $\mathrm{vw}:$ very weak, - : not observed.

$\begin{array}{lll} & - & - \\ \mathrm{C}_{12} & \mathrm{VS} & \mathrm{m} \\ \mathrm{C}_{8} & \mathrm{VS} & \mathrm{vw} \\ \mathrm{C}_{5} & \mathrm{~S} & \mathrm{vw} \\ \mathrm{C}_{4} & \mathrm{~S} & \mathrm{w} \\ \mathrm{C}_{3} & \mathrm{~S} & \mathrm{w} \\ \mathrm{C}_{2} & \mathrm{~m} & \mathrm{w}\end{array}$

-
vw
w
vw
vw

$\begin{array}{ll}\text { w } & - \\ \text { w } & - \\ \text { vw } & - \\ \text { vw } & \text { vw } \\ \text { vw } & -\end{array}$


One can notice that :

- the intensity of the (10) reflection decreases with decreasing chain length. (This was estimated by comparing the intensity of the (10) reflection with the other reflections of the same compound and also by directly comparing the (10) reflections of the different compounds, assuming that the sample volumes were similar and that all diffraction conditions were nearly identical) ;

- the (11) reflection of compound $\mathrm{C}_{12}$ is still fairly strong while the (21) reflection is stronger than the $(20)$ one ;

- the (11) reflection of compound $\mathrm{C}_{8}$ is quite weak and its (20) reflection is missing whereas its (21) reflection can be detected;

- for compound $\mathrm{C}_{5}$, an intensity inversion occurs between reflections (11) and (20);

- in the case of compounds $\mathrm{C}_{4}$ and $\mathrm{C}_{3}$, no intensity inversion can be detected, the reflection intensities regularly decrease. In the case of compound $\mathrm{C}_{3}$, the (30) reflection could even be observed. This implies that the Debye-Waller factor is not very large and somewhat smaller than that of the usual $D_{h}$ phases [10];

- at last, for compound $\mathrm{C}_{2}$, the (10) reflection is rather weak compared to the other members of the series and the (21) reflection is as strong as the (20) one.

2.3 Model of THE MOlecular ORganization IN THE $\mathrm{D}_{\mathrm{h}}$ PHASE. - In a next step, we can try to qualitatively account for these intensities by assuming a simple structural model of the mesophase.

Let us describe the hexagonal mesophase by a two component system : the molten aliphatic medium of low and uniform electronic density; the aromatic cores and the anions as a medium of larger and uniform electronic density. As a result of diffraction theory [14], we can assign a value $\rho_{0}=0$ to the electronic density of the aliphatic medium without altering the calculated reflection intensities. In other words, the reflection intensities only depend on the contrast between the electronic densities of the two media. Furthermore, since we do not measure absolute intensities, we have only access to the relative intensities of the reflections for a given compound. Consequently, we can assign an arbitrary value $\rho_{1}=1$ to the electronic density of the second medium. This second medium consists of discs of constant radius $R$ for all compounds. These discs are located at the corners of the unit cell. Under these assumptions, one can compute the intensities of the different orders of reflection of the 2D hexagonal lattices :

The structure factor of a reflection of wavevector $s$ is classically given by $[15,16]$ :

$$
F(s)=2 \pi \int_{0}^{R} J_{0}(2 \pi r s) r \mathrm{~d} r
$$

where $J_{0}$ is the Bessel function of zero order and the corresponding intensity is :

$$
I(s)=K \frac{J_{1}^{2}(2 \pi R s)}{s^{2}}
$$

where $K$ is a constant factor.

This function (for $R=7.75 \AA$ ) is represented in figures $8 \mathrm{a}$ and $8 \mathrm{~b}$ (with a scale expansion by a factor 2 compared to Fig. 8a). The calculated intensities of the $2 \mathrm{D}$ lattice reflections for a given compound are represented by the values of this function for their scattering vectors $s$.

Looking at figure 8 , the following remarks can be made : qualitatively, the model accounts for the relative decrease of the (10) reflection with decreasing chain length. The (11) reflection of compound $\mathrm{C}_{12}$ is appreciable because it still falls on the side of the first 


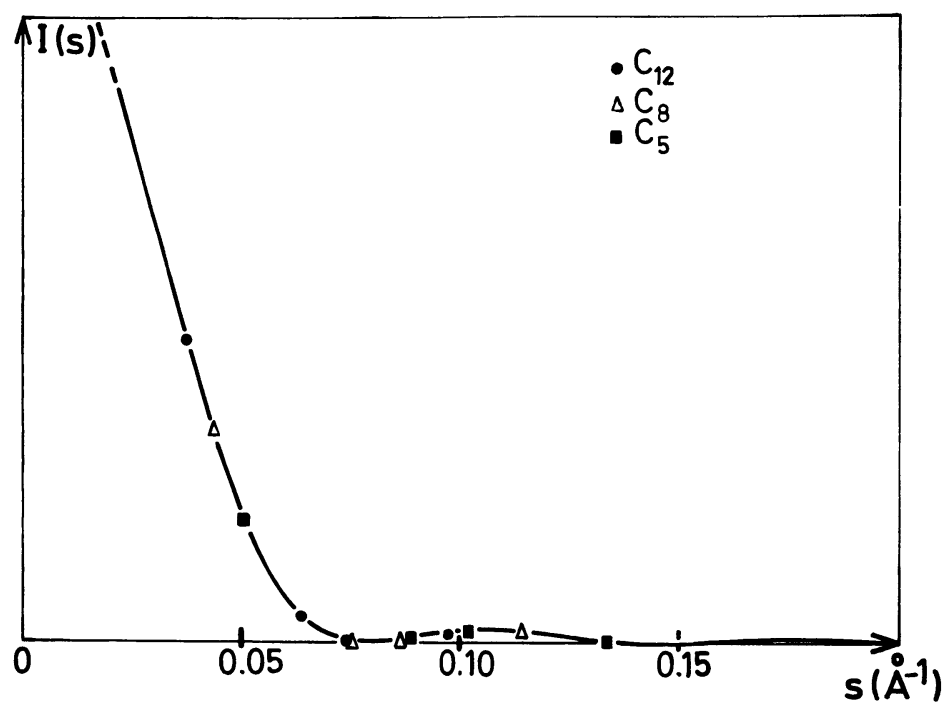

a)

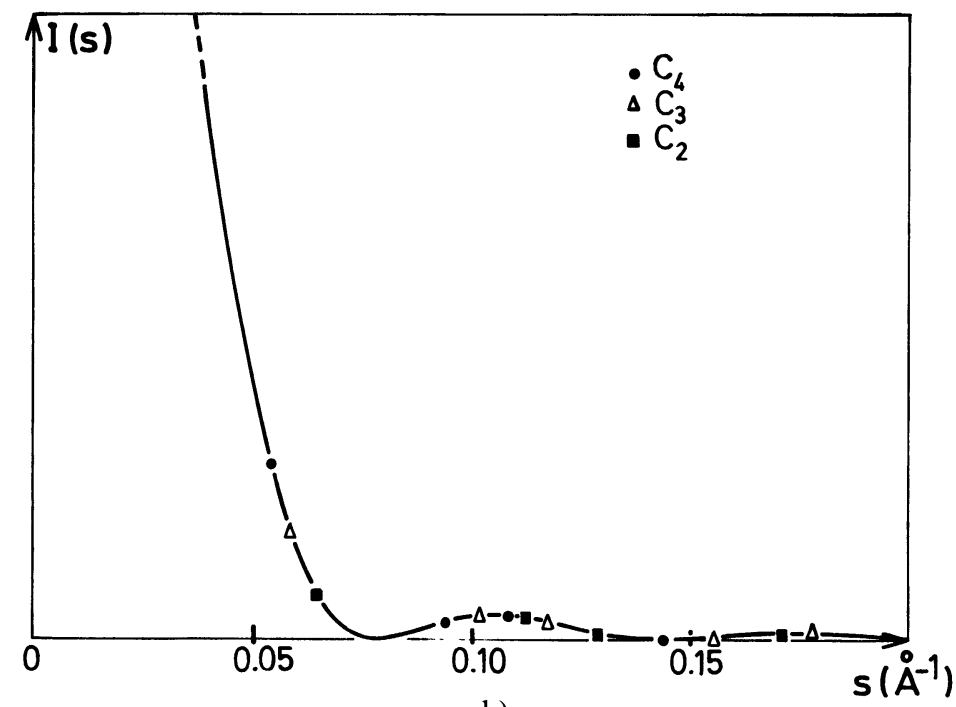

b)

Fig. 8. - (a) Function $I(s)=K J_{1}^{2}(2 \pi R s) / s^{2}$ for $R=7.75 \AA$ and its values for the different wavevectors of the $2 \mathrm{D}$ lattice reflections for compounds $\mathrm{C}_{12}, \mathrm{C}_{8}$ and $\mathrm{C}_{5}$. (b) Same function $I(s)$ (expanded by a scale factor 2 compared to Fig. 8a) and its values for the different wavevectors of the $2 \mathrm{D}$ lattice reflections for compounds $\mathrm{C}_{4}, \mathrm{C}_{3}$ and $\mathrm{C}_{2}$.

maximum of the function. The (21) reflection is stronger than the (20) one because the (20) reflection is close to the first minimum of the function. For compound $\mathrm{C}_{8}$, the (11) reflection is very weak and the (20) reflection cannot be seen because they are located close to the first minimum of the function. However, the (21) reflection can indeed be detected. For compound $\mathrm{C}_{5}$, the (11) reflection still falls in the first minimum of the function so that it is weaker than the (20) one. As observed experimentally, the reflection intensities of compounds $\mathrm{C}_{4}$ and $\mathrm{C}_{3}$ decrease regularly showing no intensity inversion. The (30) reflection 
of compound $\mathrm{C}_{3}$ is located on top of the third maximum of the function and therefore it can be detected. Finally, for compound $\mathrm{C}_{2}$, the (10) reflection is not so strong compared to the others because it falls close to the first minimum of the function and both reflections (20) and (21) are comparable because they fall on each side of the second minimum.

Therefore, this simple model is in fair agreement with the experiments for a core radius of $R=7.75 \pm 0.25 \AA$. This value is slightly larger than the core radius measured on Dreiding stereomodels $\left(\approx 7 \pm 0.5 \AA\right.$ ). This could be due to the presence of the $\mathrm{BF}_{4}^{-}$anions which should be found close to the aromatic cores in order to minimize the electrostatic energy. Since no reflections of wavevector $s>0.18 \AA^{-1}$ can be detected, the aliphatic and the aromatic media must be somewhat disordered. This explains why our simple model with only two electronic levels agrees fairly well with the experimental results. This is the direct consequence of the liquid crystalline character of the $D_{h}$ phase.

As far as the $\mathrm{BF}_{4}^{-}$anions are concerned, the only information that we could obtain is that they seem to be located close to the aromatic cores. Furthermore, if they were located and ordered in special positions of the unit cell, we possibly might have observed their influence on the 2D lattice reflection intensities.

At this point, we found it interesting to obtain more information on the $\mathrm{BF}_{4}^{-}$anions because their role in the mesophase formation must be important: in order to obtain a columnar mesophase, it is necessary that the molecules tend to stack themselves in columns (separating the aromatic and aliphatic mediums) and also that molecules of neighbouring columns should not be 3-dimensionnally correlated. This last point is usually assured by the presence of long aliphatic chains around the aromatic core. However, in the case of the short chain substituents $\left(\mathrm{C}_{2}-\mathrm{C}_{5}\right)$, it is quite possible that the $\mathrm{BF}_{4}^{-}$anions, thanks to their quasispherical shape, also tend to decorrelate the neighbouring columns.

\section{NMR.}

In order to obtain specific information on the location and dynamic behaviour of the $\mathrm{BF}_{4}^{-}$anions, preliminary ${ }^{19} \mathrm{~F}$ NMR experiments have been done on compounds $\mathrm{C}_{4}, \mathrm{C}_{5}, \mathrm{C}_{8}$ and $\mathrm{C}_{12}$ between room temperature and about $200{ }^{\circ} \mathrm{C}$. In that temperature range, compounds $\mathrm{C}_{5}, \mathrm{C}_{8}$ and $\mathrm{C}_{12}$ are in the columnar mesophase $\mathrm{D}_{\mathrm{h}}$, whereas $\mathrm{C}_{4}$ becomes mesomorphic at a temperature higher than $140^{\circ} \mathrm{C}$. Given the temperature range required to attain the $D_{h}$ phase, and the duration of experiments, thermal decomposition of samples $C_{2}$ and $C_{3}$ did not allow to achieve satisfactory experiments in these compounds at the moment.

3.1 EXPERIMENTAL. - A Brüker CXP90 pulsed spectrometer, together with a conventional electromagnet, has been used to measure ${ }^{19} \mathrm{~F}$ resonance line width as a function of temperature, at a Larmor frequency $80 \mathrm{MHz}$. Temperature is regulated (to $\pm 1^{\circ} \mathrm{C}$ ) by an heated air flow. The resonance line is obtained by fast Fourier transforming the averaged free induction decay observed after a single $\pi / 2$ pulse, of length about 2 to $3 \mu$ s. The recovery time is about $5 \mu \mathrm{s}$, the digitization rate is $1 \mathrm{MHz}$. These conditions are adequate to observe the resonance line, which is about $15 \mathrm{kHz}$ wide at most. The accumulation number is of the order 2000 to 5000 . Measures of the longitudinal relaxation time $T_{1}$ versus temperature have also been achieved; only a qualitative variation can be described at the moment.

3.2 Results. - In compounds $\mathrm{C}_{4}, \mathrm{C}_{5}$ and $\mathrm{C}_{8}$, a roughly Gaussian resonance line, with a half-height line width of the order 13 to $15 \mathrm{kHz}$, is observed at room temperature. Then, a steep decrease of the line width between, say, $25^{\circ} \mathrm{C}$ and $125^{\circ} \mathrm{C}$ clearly indicates the onset of $\mathrm{BF}_{4}^{-}$motion (Fig. 9). This motional narrowing is not associated with a $\mathrm{K} \rightarrow \mathbf{M}$ transition : indeed, for compounds $\mathrm{C}_{5}$ and $\mathrm{C}_{8}$, it takes place within the mesophase stability domain, 


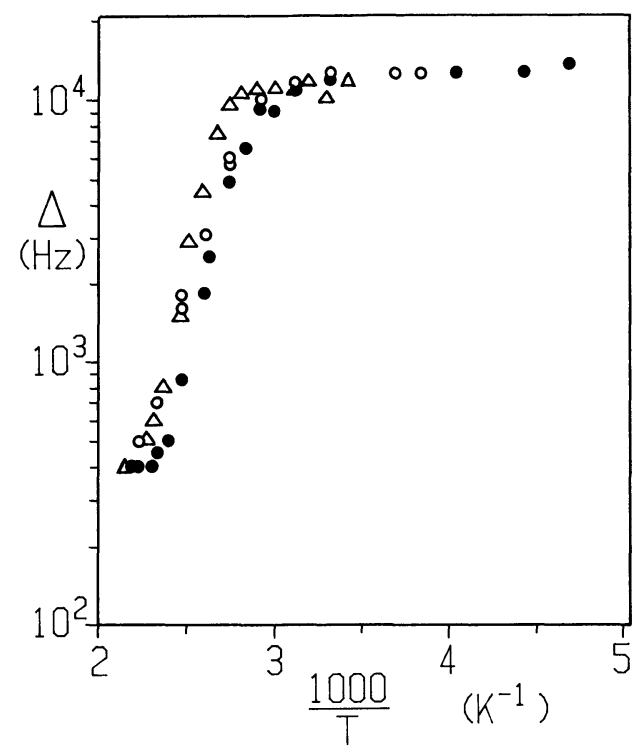

Fig. 9. - Half-height line width $\Delta$ of the ${ }^{19} \mathrm{~F}$ nuclear magnetic resonance line in the $\mathrm{BF}_{4}^{-}$anions, as a function of temperature : $(\mathrm{O})$ compound $\mathrm{C}_{8} ;(\bullet)$ compound $\mathrm{C}_{5} ;(\Delta)$ compound $\mathrm{C}_{4}$.

whereas for compound $\mathrm{C}_{4}$, it becomes complete within the crystalline phase, even before attaining the mesophase. The reduction factor ( $11 / 2$ decade) is a measure of the motional averaging achieved. Remarkably, this behaviour is roughly the same for compounds $\mathrm{C}_{4}$ to $\mathrm{C}_{8}$, i.e. for different chain lengths (Fig. 9). However, for compound $C_{12}$, the resonance line in the same temperature range $\left(50<T<150^{\circ} \mathrm{C}\right)$ is much narrower, indicating a more efficient motional averaging. In this latter compound, it is also worth noting that the line shape is significantly altered between $35^{\circ} \mathrm{C}$ and $55^{\circ} \mathrm{C}$ (Fig. 10). This coincides with a transition (possibly a $\mathrm{K} \rightarrow \mathrm{M}$ transition) already mentioned [6]. The line shape which is observed under $35^{\circ} \mathrm{C}$ (qualitatively similar to that one called "super-Lorentzian " [17]), might thus be associated with this crystalline phase, in contrast to the Gaussian line shape, related to the

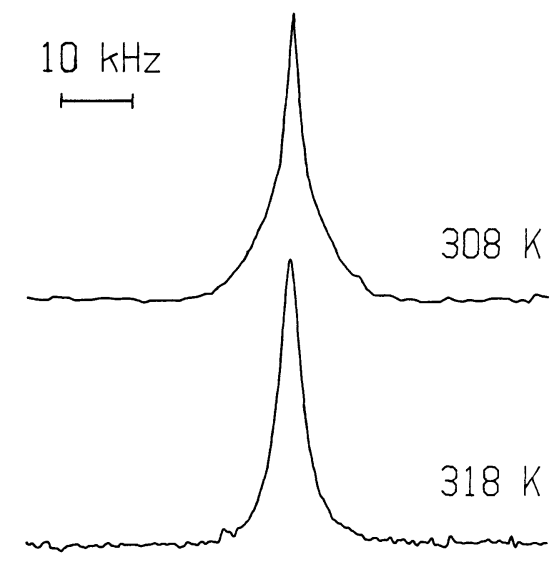

Fig. 10. $-{ }^{19} \mathrm{~F}$ nuclear magnetic resonance lines in the $\mathrm{BF}_{4}^{-}$anions, for the $\mathrm{C}_{12}$ compound: at a temperature $308 \mathrm{~K}$, the line shape is similar to a "super-Lorentzian " one; at $318 \mathrm{~K}$, it has become nearly Gaussian. 
columnar mesophase. Note also that $T_{1}$ increases with temperature. This suggests that the longitudinal relaxation is not limited by the motional frequency, indicating that the motions contributing to longitudinal relaxation are faster than the Larmor frequency [18].

3.3 DisCUSSION. - A comprehensive understanding of the observed behaviour lies upon the determination of the interactions contributing to the line width on the one hand, and the motions which may average these interactions, on the other hand. The most likely contributions arise from :

- homonuclear $\left({ }^{19} \mathrm{~F}^{19} \mathrm{~F}\right)$ and heteronuclear $\left({ }^{19} \mathrm{~F}-{ }^{11} \mathrm{~B}\right)$ intramolecular (inside a $\mathrm{BF}_{4}^{-}$ anion) dipolar couplings.

- intermolecular $\left({ }^{19} \mathrm{~F}-{ }^{1} \mathrm{H}\right)$ dipolar couplings, involving protons either in the aromatic core or in the alcoxy chains.

- intermolecular $\left({ }^{19} \mathrm{~F}-{ }^{19} \mathrm{~F}\right)$ couplings have been neglected in a first approach.

Then, the different types of motion which may be distinguished are the following:

a) first, the $\mathrm{BF}_{4}^{-}$anion may be affixed to the heteroaromatic core. From steric and electrostatic considerations, a likely fixation site should be close to the oxygene atom in the pyrylium ring (see the molecule formula in the Introduction). In that case, the only motion undergone by the anion is the eventual rotation of the ionic pair around the local director $n$, which is the axis of the column. Additional averaging might also come from fluctuations of the molecular axis around the axis of the columns. Note also that another important possibility with axial symmetry may be a "ball-bearing motion " of the anions relative to the cations;

b) then, the $\mathrm{BF}_{4}^{-}$anion, yet trapped in a fixation site, may undergo fast reorientations, due to its quasi spherical shape. Compared to the situation described above, the effect would be to average the intramolecular interactions, but not the intermolecular ones.

These orientational motions may be distinguished according to their symmetry, which is either axial (in case a), or spherical (in case b) ;

c) last, the $\mathrm{BF}_{4}^{-}$may undergo translational diffusion, by various processes : site-to-site jump, fast dynamic exchange between different solvation sites (near the core $v s$. inside the aliphatic region).

The Van Vleck formula can be used to give an estimate of the static line width (or equivalently, the second moment $M_{2}$ ) [18]. Assuming that the line width is dominated by nearest-neighbour interactions, this formula reduces to :

$$
M_{2}=\sum_{\alpha} M_{2}^{(\alpha)}
$$

where :

$$
M_{2}^{(\alpha)}=\hbar^{2} \gamma_{\mathrm{F}}^{2} \gamma_{\alpha}^{2} I_{\alpha}\left(I_{\alpha}+1\right) A_{\alpha} \sum_{k} \frac{P_{2}^{2}\left(\cos \theta_{k}\right)}{R_{k}^{6}}
$$

The subscript $\alpha$ denotes the 3 different nuclei coupled with ${ }^{19} \mathrm{~F}\left({ }^{19} \mathrm{~F}\right.$ : spin $I_{1}=$ $\left.1 / 2, A_{1}=3 ;{ }^{11} \mathrm{~B}: \operatorname{spin} I_{2}=3 / 2, A_{2}=4 / 3\right)$ [19]; $\gamma_{\alpha}$ is the corresponding gyromagnetic ratio. The summation over $k$ extends to all internuclear vectors for each species coupled with ${ }^{19} \mathrm{~F}$. In the intramolecular term $M_{2}^{(2)}$, the internuclear distance $R_{k}$ has been estimated by use of covalent atomic radii : $R_{2}=r_{\mathrm{F}}+r_{\mathrm{B}}=1.52 \AA$; in $M_{2}^{(1)}$, use has been made of the tetrahedral structure of the $\mathrm{BF}_{4}^{-}$anion (see Fig. 11a) which leads to $R_{1}=2.48 \AA$ [13]. The vector between the interacting nuclei makes an angle $\theta_{k}$ with the magnetic field. The contribution 

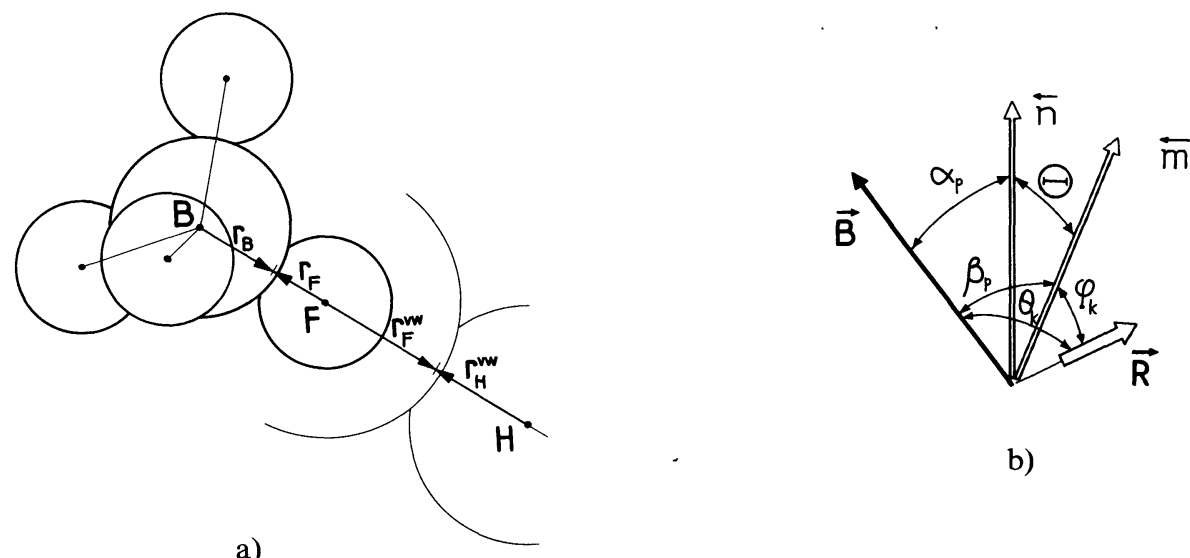

b)

Fig. 11. - (a) The tetrahedral configuration of a $\mathrm{BF}_{4}^{-}$anion, showing the different internuclear vectors $R_{k}$. (b) The different angular coordinates used to define the orientation of the internuclear vector $R$ and of the molecular axis $m$, with respect to the magnetic field $B$ or the local director $n$ (which is the axis of the column).

$M_{2}^{(3)}$ associated with the (intermolecular) ${ }^{19} \mathrm{~F}-{ }^{1} \mathrm{H}$ couplings is more difficult to estimate. An upper limit can be given, assuming that each fluor nucleus comes close to one proton (see Fig. 11a) (at a distance $R_{3}=r_{\mathrm{F}}^{\mathrm{vw}}+r_{\mathrm{H}}^{\mathrm{vw}}=2.55 \AA$, where $r_{\alpha}^{\mathrm{vw}}$ are Pauling's Van der Waals radii). Even in this idealized situation, $M_{2}^{(3)}$ remains rather weak with respect to the intramolecular contributions $\left(M_{2}^{(3)}=M_{2}^{(1)} / 6=M_{2}^{(2)} / 12\right)$.

If a random distribution of the molecular axis throughout the sample is assumed (powder situation), the $P_{2}^{2}\left(\cos \theta_{k}\right)$ term is to be averaged over all directions, which leads to a factor $1 / 5$ [20]. The half-height line width is then given by (assuming that the line shape is Gaussian) :

$$
\Delta=2.355 M_{2}^{1 / 2} \text {. }
$$

This estimation for the static line width may probably be considered as a lower limit, insofar as only nearest neighbour interactions have been taken into account. The half-height line width estimated in this manner amounts to about 30 to $35 \mathrm{kHz}$, which exceeds the experimental one by a factor 2.5. Therefore, some motion, which averages part of the interactions, must be envisaged. Let us recall that $\mathrm{X}$-ray diffraction experiments do not give access to the dynamics.

Fast rotation around the columnar axis. - Several authors have carried on deuterium NMR experiments (using specific deuteriation) on various non-ionic discotic compounds (especially, uniaxial columnar mesophases of compounds based on a triphenylene core substituted with 6 alkyl chains) [21-23]. These studies indicated that rotation of the molecules around their axis, though much slower than in usual (nematic) liquid crystal, remains fast on the ${ }^{2} \mathrm{H}$ NMR time scale (i.e., faster than $10^{-6} \mathrm{~s}$ ), leading to axially symmetric average interaction tensors. This is mainly demonstrated by the presence of sharp doublets, which allow to measure a dynamic order parameter with respect to the local director $n$, as in usual nematic compounds. This order parameter is found to be high $(\approx 0.9)$ and remains almost constant over a wide temperature range [21]. ${ }^{13} \mathrm{C}$ and ${ }^{1} \mathrm{H}$ NMR studies were also published and lead to similar conclusions [24, 25]. Such a dynamic behaviour has not yet been proved in our ionic systems. However, it seems reasonable to examine such a possibility, and to estimate the eventual 
effect of such a rotation on the line width. This is done by replacing the $P_{2}\left(\cos \theta_{k}\right)$ angular functions in the dipolar Hamiltonian, and thus in the expression for the second moment, by temporal averages of these functions over fast molecular rotations. First, if the molecule undergoes fast rotation around its axis, one may write (see Fig. 11b) :

$$
\left.\left.\overline{P_{2}\left(\cos \theta_{k}\right.}\right)=\overline{P_{2}\left(\cos \beta_{p}\right.}\right) P_{2}\left(\cos \varphi_{k}\right) \text {. }
$$

If, additionally, the molecular axis fluctuates rapidly around the local director $n$ (the axis of the column), then :

$$
\left.\overline{P_{2}\left(\cos \beta_{p}\right.}\right)=\overline{P_{2}(\cos \Theta)} P_{2}\left(\cos \alpha_{p}\right)
$$

where $P_{2}(\cos \Theta)$ is the orientational order parameter $S$ of, say, the heteroaromatic cations with respect to $n$. Finally, the resulting second moment is given by:

$$
M_{2}=S^{2}\left(\sum_{p} P_{2}\left(\cos \alpha_{p}\right)\right)^{2} M_{2}^{0}
$$

where $M_{2}^{0}$ is the static second moment estimated above, and the summation between brackets (replaced by integration over all directions) amounts to 0.385 . The molecular order parameter $S$ will be taken equal to 0.9 , which is consistent with $\mathrm{X}$-ray diffraction patterns and results in similar systems [21]. So the effect is a decrease of the line width by a factor of about 0.35 . The resulting half-height line width therefore lies between 11 and $13 \mathrm{kHz}$, which is in fair agreement with the observed one (at low temperature).

Thus, the observed line width (at low temperature) may be accounted for by a rotation around the columnar axis. This averaging effect may be simply viewed as due to motion of the anions inside a potential with cylindrical symmetry. In the mesomorphic compounds $\mathrm{C}_{5}$ and $\mathrm{C}_{8}$, this behaviour is consistent with the assumption that the $\mathrm{BF}_{4}^{-}$anions are affixed to the heteroaromatic cations, whereas in the $\mathrm{C}_{4}$ (crystalline) it is necessary to suppose reorientational motions of the anions relative to the cations (" ball-bearing motions ", possibly including also site-to-site diffusion).

Spherical reorientation of the $\mathrm{BF}_{4}^{-}$anions. - The observed narrowing (Fig. 9) remains to be discussed. The onset of fast (spherical) rotation of the $\mathrm{BF}_{4}^{-}$may be responsible for it. The net effect would be to average intramolecular interactions (F-F and F-B couplings), as soon as the rotation becomes fast enough (faster than the inverse line width, i.e. than $10^{-4} \mathrm{~s}$ ). In that case, the residual intermolecular interaction may be approximated by the first term of a twocenter expansion of the spherical harmonics $P_{2}\left(\cos \theta_{k}\right)$ [26]. In this approximation, as a result of fast $\mathrm{BF}_{4}^{-}$rotation, the $4{ }^{19} \mathrm{~F}$ nuclei behave as though they were located at the center of the anion. The average H-F distance is thus $R=4 \AA$. This would lead to a residual line width of about 1.75 to $1.85 \mathrm{kHz}$ (in the absence of fast rotation of the molecules around $n$ ), or 0.54 to $0.57 \mathrm{kHz}$ (in the more realistic case of fast rotation). This later estimation is consistent with the observed line width of 0.4 to $0.5 \mathrm{kHz}$ at high temperature.

Note that it is yet assumed that some interionic interactions are not averaged. A further average of theses interactions would lead to an additional narrowing of the resonance line, which does not seem to be observed in the temperature range investigated. Such an average would result from diffusion of the $\mathrm{BF}_{4}^{-}$anions, or from exchange between two solvation sites (one intercalated inside the columns, one in the more disordered aliphatic region) [27-29].

However, a precise assignment of the observed motions would need additional experiments. Specifically, ${ }^{2} \mathrm{H}$ NMR experiments (which imply specific deuteriation) and/or diffusion coefficient measurements should help to discriminate translational and rotational motions. 


\section{Conclusions.}

Triaryl-2,4,6 pyrylium tetrafluoroborates substituted with 6 short alcoxy chains $(n=2,3)$ exhibit $D_{h}$ columnar mesophases over a fairly wide range of temperature, despite the shortness of the chains. The molecular organization of this mesophase $D_{h}$ is similar to that of the $D_{h}$ phase displayed by the other members of the series. It seems that decreasing the chain length only reduces the mesophase temperature domain of stability and shifts it towards higher temperatures without altering the molecular organization. The stability of such columnar mesophases implies that neighbouring columns be discorrelated. Disordered (or " molten ») alcoxy chains are usually assumed to play this role. This is however questionable in the case of chains as short as $n=2$ or $3 .{ }^{19} \mathrm{~F}$ NMR experiments performed on compounds $\mathrm{C}_{4}$ to $\mathrm{C}_{12}$ have shown that orientational motions of the $\mathrm{BF}_{4}^{-}$anions appear within the mesophase stability domain. The experiments suggest that the anions undergo axial rotation (around the axis of the columns and/or around parallel axis going through their own centers) and that, in the mesophase, the rotation of the anions becomes more and more spherical as temperature increases. However, a precise assignment of the observed motions would need additional experiments. This dynamic behaviour of the anions established for compounds $\mathrm{C}_{5}$ to $\mathrm{C}_{12}$, suggests that, in the case of the short chain compounds $\mathrm{C}_{2}$ and $\mathrm{C}_{3}$, these anions may play an active role in discorrelating neighbouring columns. It is worth noting that in the $\mathrm{C}_{4}$ compound, the mesophase probably appears when the motional averaging has become effective $\left(T>140^{\circ} \mathrm{C}\right)$.



Fig. 12. - Scheme of the relative disposition of the $\mathrm{BF}_{4}^{-}$anion and the cationic core. Only one conformer has been represented. The first circle of radius $7 \AA$ shows the size of the core measured on Dreiding stereomodels. The second one of radius $7.75 \AA$ represents the limit of the region of larger electron density. The anion is on average located between these two circles but is still free to undergo spatial and orientational fluctuations (see text). (The scheme is not made to scale.) 
In that respect, some information may be drawn from the X-ray analysis : the radius of the dense part of the column as derived from the fit of the experimental intensities of the 2D lattice reflections is $R=7.75 \AA$ which is larger than the $7 \AA$ measured on molecular models or obtained thanks to the curve $V_{\mathrm{c}}=f(n)$ (Fig. 7). This apparent discrepancy (for all compounds $\mathrm{C}_{2}$ to $\mathrm{C}_{12}$ ) reveals that the $\mathrm{BF}_{4}^{-}$anions are located close to the aromatic cores and that the first $\mathrm{O}-\mathrm{CH}_{2}$ links are still «crystallized» - i.e. : the $\mathrm{CH}_{2}$ groups are rather well spatially localized. This region of larger electron density is represented in figure 12. Its limit is a circle of radius $7.75 \AA$ which contains the aromatic core and the first $\mathbf{C H}_{2}$ groups. Indeed, compound $\mathrm{C}_{1}$ is not mesomorphic. In the case of the other compounds $\mathrm{C}_{2}-\mathrm{C}_{12}$, at a distance $R=7.75 \AA$, starts a disordered region of lower electronic density which may be some $8 \AA$ wide in the case of $\mathrm{C}_{12}$ but only $1.25 \AA$ wide in the case of $\mathrm{C}_{2}$. Thus, the liquid or " molten " region starts from the second methylene group of the alcoxy chains (Fig. 12).

At this point, we may recall the sharp decrease in the $\mathrm{K}-\mathrm{D}_{\mathrm{h}}$ transition temperature from $167^{\circ} \mathrm{C}$ for compound $\mathrm{C}_{3}$ to room temperature for compound $\mathrm{C}_{5}$. It seems that two different mechanisms may be responsible for the mesophase formation. The first one (for compounds $\mathrm{C}_{5}-\mathrm{C}_{12}$ ) is the usual " molten " state of the aliphatic chains which occurs at low temperatures. The second one describes the case of compounds $\mathrm{C}_{2}$ and $\mathrm{C}_{3}$ : it may be a combination of the onset of the spherical rotation of the $\mathrm{BF}_{4}^{-}$anions which was detected by NMR for compounds $\mathrm{C}_{5}-\mathrm{C}_{12}$ at high temperature and the disordered movements of the $\mathrm{CH}_{2}-\mathrm{CH}_{2}$ links which are more free to explore space than the first $\mathrm{O}-\mathrm{CH}_{2}$ one. To some extent, in the case of compound $\mathrm{C}_{2}$, the $\mathrm{BF}_{4}^{-}$anions and the $\mathrm{CH}_{2}-\mathrm{CH}_{3}$ links play the role of ballbearings which discorrelate the cationic columns. Finally, the intermediate case of compound $\mathrm{C}_{4}$ may represent a situation of crossover between the two regimes.

\section{Acknowledgments.}

We thank the referees for their comments, which allowed us to greatly improve the presentation of the paper.

\section{References}

[1] Destrade C., Foucher P., Gasparoux H., N'Guyen H. T., Levelut A. M. and Malthete J., Mol. Cryst. Liq. Cryst. 106 (1984) 121.

[2] Harada Y. and Matsunaga Y., Bull. Chem. Soc. Jpn 61 (1988) 2739.

[3] Ohta K., Murok H., Takagi A., Hatada K. I., Ema H., Yamamoto I. and Matsuzaki K., Mol. Cryst. Liq. Cryst. 140 (1986) 131.

[4] Bunning J. D., Goodby J. W., Gray G. W. and Lydon J. E., Liquid Crystals of one and two dimensional order, Eds. W. Helfrich and G. Heppke (Springer-Verlag) 1980, p. 397.

[5] Kohne B., Praefcke K. and Billard J., Z. Naturforsch. 41b (1986) 1036.

[6] Davidson P., Jallabert C., Levelut A. M., Strzelecka H. and Veber M., Liq. Cryst. 3 (1988) 133.

[7] Strzelecka H., Jallabert C. and Veber M., Mol. Cryst. Liq. Cryst. 156 (1988) 355.

[8] Strzelecka H., Jallabert C., Veber M., Davidson P. and Levelut A. M., Mol. Cryst. Liq. Cryst. 161 (1988) 395.

[9] Veber M., Davidson P., Jallabert C., Levelut A. M. and Strzelecka H., Mol. Cryst. Liq. Cryst. Lett. 5 (1987) 1.

[10] Levelut A. M., J. Chim. Phys. 80 (1983) 149.

[11] Guillon D., Skoulios A., Piechoki C., Simon J. and Weber P., Mol. Cryst. Liq. Cryst. 100 (1983) 275. 
[12] Levelut A. M., Malthete J., Destrade C. and Tinh N. H., Liq. Cryst. 2 (1987) 877.

[13] Kistenmacher T. J., Mol. Cryst. Liq. Cryst. 136 (1986) 361.

[14] Guinier A., $X$ ray diffraction in crystals, imperfect crystals and amorphous bodies (W. H. Frieman and Co., San Francisco) 1965.

[15] Levelut A. M., J. Phys. Lett. France 40 (1979) L81.

[16] Weber P., Guillon D., Skoulios A. and Miller R. D., J. Phys. France 50 (1989) 793.

[17] This term has been introduced in anisotropic fluid systems, to describe a resonance line resulting from an interaction whose strength follows a Gaussian distribution, and randomly oriented. See : Wenerström H., Chem. Phys. Lett. 18 (1973) 41.

[18] Abragam A., The Principles of Nuclear Magnetism (Clarendon Press, Oxford) 1961.

[19] The effect of the presence of ${ }^{10} \mathrm{~B}$ isotope (spin 3, natural abundance $20 \%$ ) has no important effect upon the line width.

[20] An additional factor of 3 is to be included in $M_{2}^{(1)}$, for each ${ }^{19} \mathrm{~F}$ nucleus has $3{ }^{19} \mathrm{~F}$ neighbours.

[21] Goldfarb D., Luz Z. and Zimmermann H., J. Phys. France 42 (1981) 1303.

[22] Dong R. Y., J. Chem. Phys. 88 (1988) 3962.

[23] Goldfarb D., Dong R. Y., Luz Z. and Zimmermann H., Mol. Phys. 54 (1985) 1185.

[24] Vilfan M., Lahajnar G., Rutar V., Blinc R. and Topic B., J. Chem. Phys. 75 (1981) 5250.

[25] Rutar V., Blinc R., Vilfan M. and Dubois J. C., J. Phys. France 43 (1982) 761.

[26] McBrierty V. J. and Douglas D. C., Phys. Rep. 63 (1980) 61.

[27] Goldfarb D., Luz Z. and Zimmermann H., J. Phys. France 43 (1982) 421.

[28] Goldfarb D., LuZ Z. and Zimmermann H., J. Phys. France 43 (1982) 1255.

[29] Goldfarb D., Luz Z. and Zimmermann H., J. Chem. Phys. 78 (1983) 7065. 